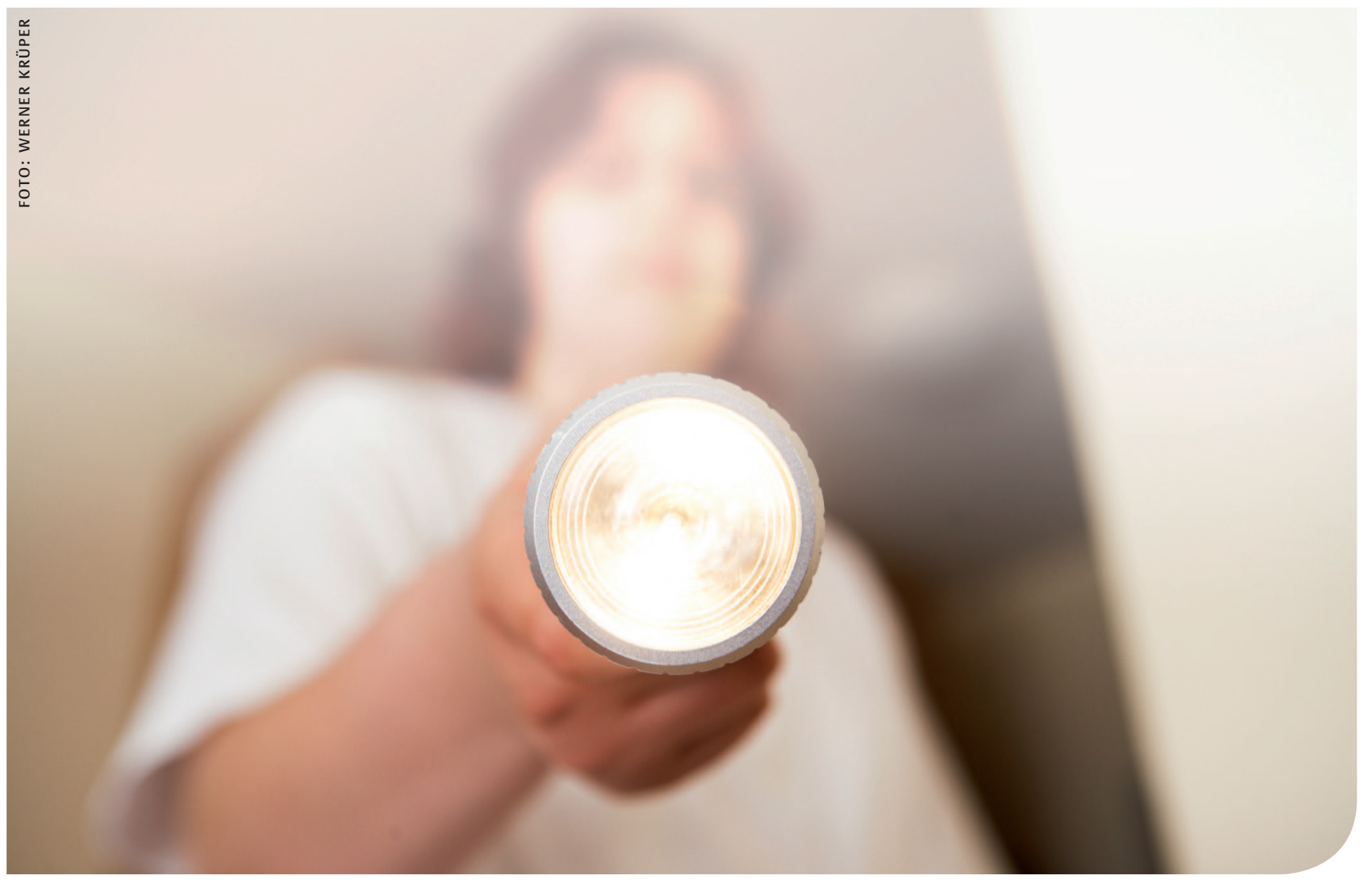

NEUE IMPULSE FÜR DIE GESTALTUNG DER PFLEGEKRAFT-PATIENT-BEZIEHUNG

\title{
Psychotraumatologie in der Bezugspflege
}

\section{Angelika Pillen}

Durch die Einführung der Bezugspflege in den meisten psychiatrischen Einrichtungen hat die Beziehungsgestaltung als Aufgabengebiet der psychiatrischen Pflege eine deutlichere Kontur gewonnen. Als Zuständige für die Pflegeplanung und die Gestaltung des Pflegeprozesses ihrer Patienten sind Bezugspflegende dazu verpflichtet, ihr jeweiliges Handeln zu begründen und die Folgen ihres Handelns systematisch zu überprüfen. Damit sind sie dazu aufgefordert, sich und anderen zu vergegenwärtigen, was sie tun und warum sie das, was sie tun, so tun, wie sie es tun.

\section{Bezugspflege in der Psychiatrie}

Die strukturelle Verankerung einer reflexiven Ebene regt dazu an, implizites Erfahrungswissen explizit und so einer systematischen Überprüfung und Abwägung zugänglich zu machen. Dies stellt hohe Anforderungen an die psychiatrische Pflege. Denn die in der Kommunikation und Interaktion mit den Patienten stattfindenden Prozesse sind oft subtil und entziehen sich einer eindeutigen Objektivierung. Die über die psychiatrische Krankheitslehre vermittelten Wahrnehmungsraster können zwar eine grobe Orientierung geben, werden der konkreten Individualität des Patienten jedoch nicht gerecht. Unter Umständen können sie sogar kontraproduktiv für die Gestaltung der Beziehung sein, denn sie engen den Blick auf den Patienten, weil sie ihn vorstrukturieren, stets ein Stück weit ein.

\section{Aktivitäten des täglichen Lebens}

Als Bezugspunkt für ihre Interventionen dienen der Pflege bekanntlich die Aktivitäten des täglichen Lebens. In der damit gegebenen Verwobenheit mit der Sphäre des Alltäglichen finden wir einen weiteren Grund dafür, dass es Pflegenden oft schwer fällt, ihre Interventionen explizit zu machen. Denn die Handlungen, die zur Bewältigung des Alltäglichen erforderlich sind, sind in ihrer Unmittelbarkeit so selbstverständlich und nah, dass es einer gewissen Mühe bedarf, sich in eine Distanz zu ihnen zu bringen. Dies ist jedoch die Voraussetzung dafür, sie wahrnehmen und explizit vergegenwärtigen zu können.

Bezugspflegende in der Psychiatrie haben die Aufgabe, die Beziehung zu den ih- 
nen zugeordneten Patienten so zu gestalten, dass eine Vertrauensbasis hergestellt wird. Damit schaffen sie die Voraussetzung für die Bearbeitung der Probleme, die den Patienten daran hindern, sein Leben so zu führen, dass er in hinreichender Weise Wohlbefinden und Zufriedenheit erfahren kann.

\section{Beziehung als Lern- und Übungsfeld}

Die Beziehungsgestaltung dient dem Ziel, den Patienten bei der Bewältigung seiner Probleme zu unterstützen bzw. dabei, einen besseren Umgang mit diesen Problemen zu finden. Zu diesem Zweck bieten Bezugspflegende ihm die Möglichkeit an, die Beziehung zu ihnen als Lern- und Übungsfeld zu nutzen. Sie unterstützen ihn bei der
Bezugspflegende sind für den Patienten zugleich Modell, Resonanzboden und Coach.
Kompetentes Handeln

in einer professionellen Beziehung

In konkreten Einzelsituationen der Praxis ist es immer wieder schwierig, zuweilen sogar unmöglich, Bezugspflege und Therapie klar voneinander abzugrenzen. In beiden Bereichen finden Prozesse statt, die therapeutische Wirkfaktoren enthalten. In der Therapie geschieht dies vorwiegend im Medium der Reflexion, in der Bezugspflege vorwiegend im Medium des Alltagshandelns, das in einem therapeutischen Prozess indessen stets mehr ist als einfaches Alltagshandeln. Da die Probleme des Patienten hier gegenwärtig werden, bietet das Alltagshandeln zahlreiche Anlässe zu ihrer Bearbeitung. PfleEntwicklung einer angemessenen Selbstund Fremdwahrnehmung und helfen ihm dabei, in der Beziehung zu sich selbst und zu anderen Menschen Achtsamkeit zu entwickeln und destruktive Impulse zu regulieren. Sie sind für ihn zugleich Modell, Resonanzboden und Coach.

Mit den soeben beschriebenen Aufgaben ist die Bezugspflege integraler Bestandteil des therapeutischen Angebotes des jeweiligen Versorgungsbereiches. Während die Therapien die Probleme des Patienten vorwiegend diskursiv und somit auf einer Metaebene angehen, begegnet die Bezugspflege den Problemen auf der Ebene des konkreten Alltagshandelns. Strategien, die in der Therapie entwickelt und geplant werden, werden in der Bezugspflege auf der Ebene des Alltagshandelns erprobt und auf ihre Tauglichkeit hin überprüft. So erfolgt mittels der Bezugspflege die Realitätsprüfung für das, was in der Therapie erarbeitet worden ist.

Therapie und Bezugspflege sind wechselseitig aufeinander verwiesen, die Therapie kommt bei Menschen, deren Fähigkeit zur Bewältigung des eigenen Lebens erheblich eingeschränkt ist, nicht ohne das Lernfeld der Bezugspflege aus, umgekehrt bedarf die Begleitung des Alltagshandelns durch die Bezugspflege der reflexiven Durchdringung der Therapie, da die Betroffenen ihren Problemen ja in diesem Bereich begegnen. gekräfte sind mit der Art und Weise, wie sie die Aktivitäten des täglichen Lebens gemeinsam mit dem Patienten gestalten, ihn bei der Nutzung seiner Ressourcen unterstützen und ihm helfen, neue Ressourcen zu entwickeln, therapeutisch wirksam. Allerdings können sie ebenso durch unbedachte Formen der Interaktion maligne Entwicklungen begünstigen.

Da die diffuse Zuständigkeit des gesamten Pflegeteams im Bezugspflegesystem zugunsten einer Individualbetreuung aufgehoben ist, wird die Beziehung zwischen der Pflegekraft und den ihr zugewiesenen Patienten sehr viel dichter. Damit erweitern sich die Möglichkeiten therapeutischer Wirksamkeit, zugleich aber auch die Risiken einer malignen Entwicklung bei ungünstigen Formen der Interaktion. Umso wichtiger wird es, dafür zu sorgen, dass die als Bezugspflegende arbeitenden Pfle- gekräfte mit den für ihre verantwortungsvolle Aufgabe erforderlichen Kompetenzen ausgestattet werden.

\section{Eine neue Sicht auf das Gegen- standsgebiet der Psychiatrie}

Es ist ein günstiger Zufall, dass die Etablierung der Bezugspflege in der Psychiatrie zeitlich mit einer anderen Entwicklung

zusammenfällt, von der wertvolle Impulse für die Kompetenzentwicklung der psychiatrischen Pflege ausgehen können.

In den letzten Jahren ist die zentrale Bedeutung von traumatischen Erfahrungen für die Entwicklung von psychischen Störungen immer mehr in den Fokus der psychiatrischen Aufmerksamkeit gerückt. In diesem Zusammenhang hat sich eine neue Form der Vermessung des psychiatrischen Gegenstandsgebietes herausgebildet.

\section{Psychotraumatologie}

Die Psychotraumatologie setzt sich mit den Formen auseinander, in denen traumatische Erfahrungen das Seelenleben und die Entwicklung der Persönlichkeit prägen bzw. verändern. Sie bietet uns damit ein erweitertes Verständnis für viele psychische Störungen und Symptome an und leistet so einen wichtigen Beitrag, die Nöte der betroffenen Patienten besser zu verstehen.

Der Fokus der Psychotraumatologie geht dabei weit über die direkt traumatischen Erlebnissen zugeordneten Krankheitsbilder hinaus. Sie eröffnet neue $\mathrm{Zu}$ gangsmöglichkeiten zu vielen psychischen Krankheiten, die bislang mit anderen Konzepten betrachtet werden.

\section{Traumatische Erfahrungen}

So wird inzwischen immer deutlicher, dass eine Reihe von psychiatrisch auffälligen Verhaltensweisen wie etwa der Substanzmissbrauch in einer beträchtlichen Anzahl von Fällen mit traumatischen Erfahrungen in Verbindung gebracht werden kann. Vor allem aber die Entwicklung einer Borderline-Persönlichkeitsstörung steht häufig in einem engen Zusammenhang mit schweren traumatischen Er-

\section{Mittels der Bezugspflege erfolgt die Realitätsprüfung fahrungen in der Kind-} für das, was in der Therapie erarbeitet worden ist. heit und kann daher als Folgeerscheinung einer

komplexen Traumatisierung angesehen werden [1, S.46ff.]

Die zunehmende Verbreitung des Ansatzes ist nicht zuletzt deswegen erfreulich, weil sie eine Rückbesinnung der Psychiatrie auf die Erfahrung in Aussicht stellt (der Begriff der Erfahrung wird hier im Sinne der Phänomenologie verwendet, die ihn von einem naturalistischen Verständnis 
Zunächst ermöglicht sie ein tieferes Verständnis für diejenigen, bei denen direkt eine posttraumatische Belastungsstörung diagnostiziert worden ist, darüber hinaus jedoch auch ein besseres Verständnis für viele andere, deren Probleme mit einer anderen Diagnose verbunden werden (für diesen Zusammenhang hat sich in den letzten Jahren der Begriff der Doppeldiagnosen eingebürgert).

Dynamik zwischen Traumaschema und traumakompensatorischem Schema

Die von der Psychotraumatologie beschriebene Dynamik zwischen Traumaschema und traumakompensatorischem Schema erlaubt es, den positiven Sinn vieler Verhaltenswei-

sen und Reaktionen zu verstehen, die ohne diesen Hintergrund als bizarr oder gestört beurteilt werden. So

Bezugspflegende helfen dem Patienten, den Bann des Schreckens zu brechen.

wird mit dem Wissen darum, dass eine Frau in ihrer Kindheit von ihrem Vater sexuell missbraucht worden ist, verständlich, warum sie das Essen verweigert, wenn Bratwurst auf dem Speiseplan steht oder Joghurt zum Dessert angeboten wird: Pflegekräfte, die in der stationären Versorgung von Patienten mit PTSD arbeiten, berichten, dass diese Aversionen häufig bei Frauen mit Missbrauchserfahrungen vorkommen.

Aufgrund ihrer Form oder Konsistenz rufen diese Nahrungsmittel die mit der traumatischen Kindheitserfahrung verbundenen Affekte des Ekels und der Abscheu wieder wach und machen diese gegenwärtig. Sie werden - um den für diese Phänomene gebräuchlichen Begriff der Psychotraumatologie zu verwenden - getriggert.

\section{Übertragungsdynamik}

Bezugspflegende werden aufgrund der besonderen Nähe, die sich zwischen ihnen und ihren Patienten entwickelt, unweigerlich mit Elementen der im Kasten 1 beschriebenen Logiken konfrontiert. Zudem besteht eine gewisse Wahrscheinlichkeit, dass sie im Rahmen der Übertragung in die Dynamik zwischen Traumaschema und kompensatorischen Abwehrbemühungen einbezogen werden. Für eine hilfreiche Gestaltung der Bezugspflege ist es aus diesem Grunde unabdingbar, dass die mit dieser Aufgabe betrauten Pflegekräfte dazu in der Lage sind, die Übertragungsdynamik zu erkennen und einen Umgang damit zu finden. Zumal dann, wenn die Traumatisierungen von anderen Menschen ausgegangen sind, ist es hier eine ständige Herausforderung, eine die gemeinsame Arbeit ermöglichende Vertrauensbasis herzustellen und zu erhalten.

\section{Pflegerische Interventionen}

Das durch eine traumatische Erfahrung beherrschte Erleben ist mit einer Reihe von Pflegeproblemen verbunden, die den Anlass für pflegerische Interventionen bilden. Eine wichtige Orientierung bildet in diesem Zusammenhang das Ziel, den Zustand

des in seinem Selbst- und Weltverständnis erschütterten Patienten zu stabilisieren. So kommt es im Rahmen des von Intrusionen bestimmten Erlebens der Wiederkehr des Traumas zu körperlichen Stressreaktionen wie Schlafstörungen oder Kopfschmerzen. Pflegerische Interventionen können hier etwa darin bestehen, gemeinsam mit dem Patienten Schlafrituale zu erarbeiten. Bei den mit Intrusionen einhergehenden psychischen Stressreaktionen wie dem Gefühl andauernden Bedrohtseins und/oder ausgeprägter Schreckhaftigkeit können die Bezugspflegenden den Betroffenen Entspannungstechniken vermitteln und deren Anwendung mit ihnen einüben. Des Weiteren können sie ihm dabei helfen, Trigger zu identifizieren und gemeinsam mit ihm Möglichkeiten erarbeiten, einen Umgang damit zu finden.

Im Rahmen des von Dissoziationen bestimmten Erlebens, das die Patienten dazu veranlasst, sich aus sozialen Kontakten zurückzuziehen und Begegnungen mit an das Trauma erinnernden Begebenheiten oder Dingen zu vermeiden, können die Bezugspflegenden Versuche unterstützen und begleiten, die Belastung dieser Begegnungen erneut auf sich zu nehmen. Bei dem häufig auftretenden Grübelzwang können sie ihm Möglichkeiten der Entlastung aufzeigen und ihm Distanzierungstechniken vermitteln. Nicht zuletzt identifizieren sie bei jedem Patienten die ihm zur Verfügung stehenden Ressourcen und unterstützen ihn dabei, diese für sich zu nutzen.

\section{Trauma}

In unbearbeiteten Zustand kann die traumatische Erfahrung bei Betroffenen zum organisierenden Zentrum ihrer Existenz werden und so ihr Leben beherrschen. Da gibt es diejenigen, die die Struktur der traumatischen Situation unbewusst immer wieder selber herstellen und sich an Versuchen abarbeiten, das Trauma auf diesem Weg zu überwinden. Diese Dynamik hat bereits Sigmund Freud als Wiederholungszwang beschrieben, allerdings gibt Freud dem eine triebtheoretische Begründung [4, S.229f].

In der Psychotraumatologie wird das Trauma ganz allgemein als Unterbrechung einer Handlung bestimmt. Im Zurückgehen zu dieser Unterbrechung bemüht sich der Traumatisierte darum, die unterbrochene Handlung zu ihrem Ende zu bringen [3]. In diesen Fällen gestaltet sich die Welt der Betroffenen als eine mit Schrecknissen und Gefahren angefüllte Welt, ihr Leben ist von katastrophischen Ereignissen bestimmt. Auf der anderen Seite finden wir diejenigen, die sich aus allen Lebensbezügen und sozialen Kontakten zurückziehen, um jede Möglichkeit zu vermeiden, erneut mit Elementen der traumatischen Erfahrung in Berührung zu kommen. Die Welt dieser Patienten ist eine sehr reduzierte Welt, in der der lebendige Austausch mit Anderen verloren gegangen ist. Das geht mit einer Abspaltung von Gefühlen einher mit der Folge, dass die Betroffenen keine Verbindung mehr zu ihren Gefühlen herstellen können. Während die zuerst Genannten sich immerzu von der traumatischen Erfahrung überflutet fühlen (Intrusion) und in der Folge in einen Zustand der Erregung geraten (Hyperarousal), haben diese den Zugang zu dieser Erfahrung erfolgreich versperrt (Dissoziation), allerdings um den Preis, sich emotional tot zu fühlen (Numbing). 


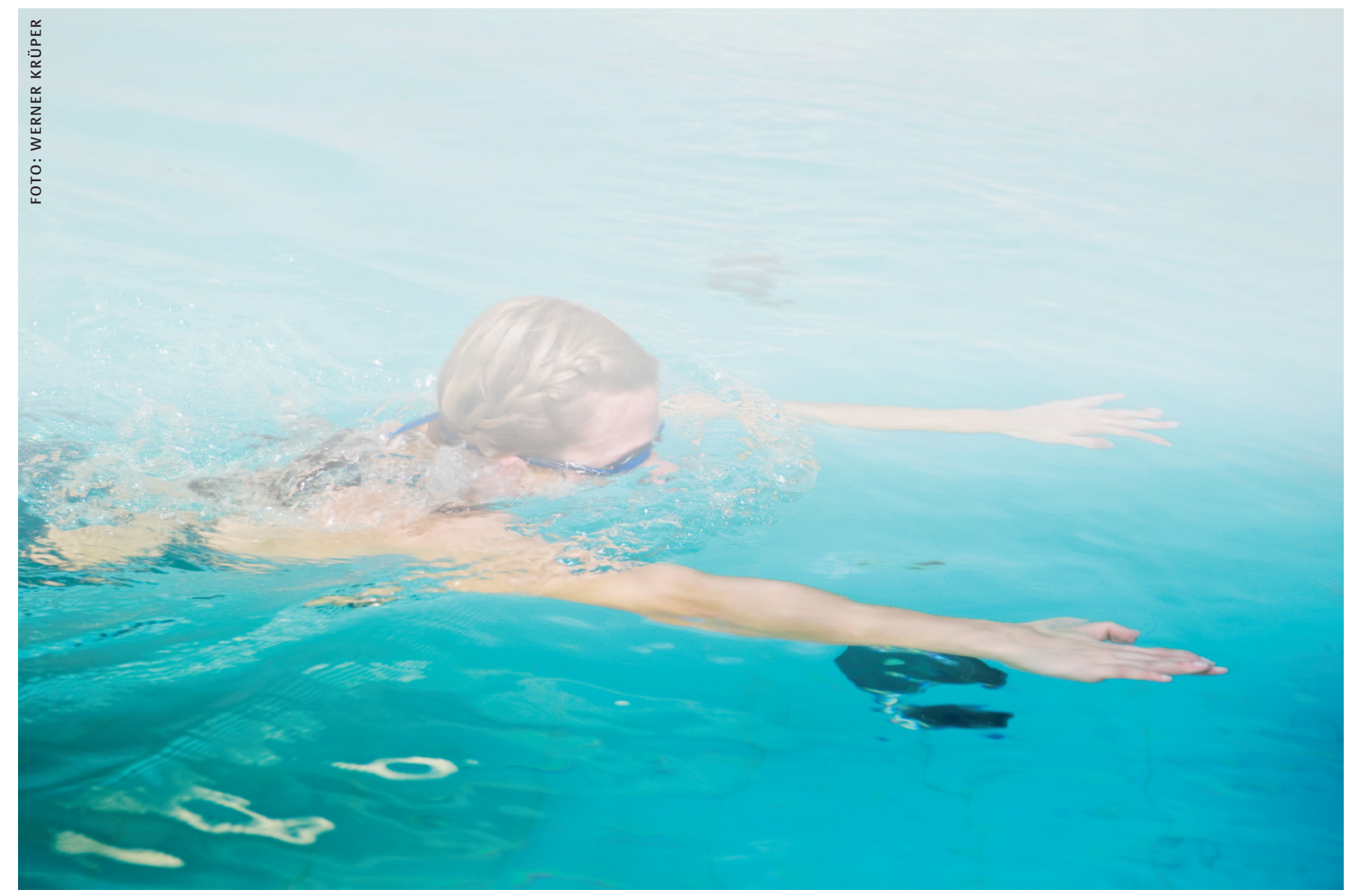

\section{Selbstwirksamkeit}

Alle Interventionen dienen dem Ziel, dem Patienten Möglichkeiten der Selbstwirksamkeit zu vermitteln. Bezugspflegende begleiten ihn bei seinen Versuchen, die verloren geglaubte und zuweilen auch wirklich verloren gegangene Kontrolle über das eigene Leben wieder zu gewinnen. Indem sie dem Patienten Techniken des Umgangs mit den Folgen der traumatischen Erfahrungen vermitteln und ihm die Gelegenheit geben, diese Techniken zu erproben und einzuüben, helfen sie ihm, den Bann des erlebten Schreckens, in dessen Zeichen sein Leben zu stehen scheint, zu brechen, und sich selbst in eine Distanz und Differenz zu diesem Schrecken zu bringen. Letztendlich dienen ihre Interventionen der Wiederherstellung von Autonomie.

\section{Fazit}

Die in der Behandlung und Pflege von Patienten mit PTSD entwickelten und erprobten Methoden können gewinnbringend in die Pflege von vielen anderen Patienten eingebracht werden, die Probleme im Bereich der
Selbstregulation haben. Dazu gehören vor allem die Patienten mit Borderline-Störung.

So erwerben Pflegekräfte mit Kompetenzen im Bereich der Psychotraumatologie viele Möglichkeiten zu gezielten Interventionen bei Patienten, deren Pflege als besonders schwierig wahrgenommen wird. In jedem Fall kann die Psychotraumatologie wertvolle Anregungen für eine gehaltvolle Gestaltung der PflegekraftPatient-Beziehung im Rahmen der Bezugspflege geben. Aus diesem Grunde sei es Pflegekräften empfohlen, sich mit dem Ansatz auseinanderzusetzen.

Das neu gegründete Alexianer- Institut für Psychotraumatologie bietet seit März 2011 (und erneut ab März 2012) eine Zusatzqualifikation Bezugspflege Psychotraumatologie an, mit der Pflegekräfte die beschriebenen Kompetenzen erwerben können. Nähere Auskünfte zu der Qualifikation erhalten Siebei den beiden Geschäftsstellen des AIfP (Alexianer-Institut für Psychotraumatologie) in Berlin und Krefeld (www.alexianer. de) oder bei der Autorin.

\section{Literatur}

1 Flatten G, Gast U. Posttraumatische Belastungsstörung. Leitlinie und Quellentext. Stuttgart: Schattauer; 2004

2 Hermann J, Die Narben der Gewalt. München: Kindler; 1993

3 Fischer G, Riedesser P. Lehrbuch der Psychotraumatologie. München: Ernst Reinhardt GmbH \& Co KG Verlag; 2009

4 Freud S. Jenseits des Lustprinzips. In: Freud S.

Studienausgabe Band 3, Psychologie des Unbewußten. Frankfurt/M.: S. Fischer Verlag; 1975

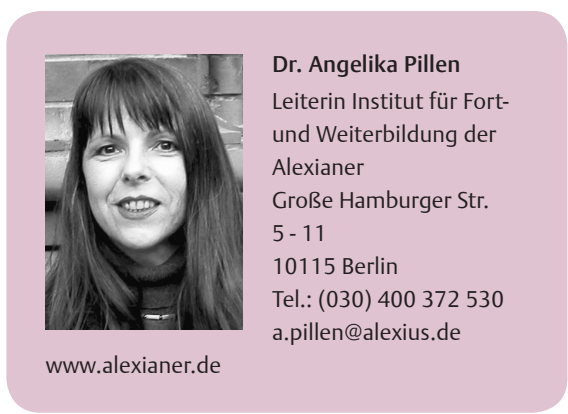

\section{Bibliografie}

DOI 10.1055/s-0031-1287910

Psych Pflege 2011; 17: 262-266

(c) Georg Thieme Verlag KG

Stuttgart · New York · ISSN 0949-1619 